A 'dark space' of 0.5 sec duration has been observed ${ }^{3}$ at the detonation front in pentolite and it was suggested that this was due to a highly ionized region which acted as a reflector and blocked the transmission of internsl light. We favour this mechanism to explain the absence of transmitted backlight in our experiments. However, Johansson and Sjölin² believe that carbon particles may form a light-absorbing screen at the surface layer of the detonation gas and they have data indicating this as a possibility. Our experiments show that the opaque reaction products from nitromethane, ostensibly free carbon, can be burned to produce clear products if the oxygen balance is adjusted by the addition of tetranitromethane to anywhere within the carbon monoxide-carbon dioxide stoichiometric limits. In line with the JohanssonSjölin explanation it is of interest to note that selfluminosity is markedly higher in those mixtures having transparent products and reaches a peak within the carbon monoxide-carbon dioxide limits. Detonation luminosity is at its lowest with pure tetranitromethane but in this case, as with oxygen-rich mixtures, the opacity may be due to nitrogen oxides.

H. Dean Mallory

R. A. Plauuson

Chemistry Division,

U.S. Naval Ordnance Test Station, China Lake, California.

${ }^{1}$ Mallory, H. D., and McEwan, W. S., Ninth Intern. Symp. Combustion, 478 (Williams and Wilkins, Baltimore, 1963).

${ }^{3}$ Johansson, C. H., and Siölin, T., Nature, 185, 523 (1960).

${ }^{3}$ Sultanoff, M., J. Photo. Sci., 5, 27 (1957).

\section{Transmission Parameters of Porous Sound Absorbers (with Particular Reference to the Flow Resistance)}

Flow resistance, being relatively easy to measure, is commonly used as a rough guide in choosing soundabsorbing materials for specific purposes. This practice is based on the tacit assumption that, when there is an alternating air-flow through the sample, the resistance encountered is identical with the flow resistance. It can be shown, however, that in the case of several materials this assumption is false, and that the acoustical resistance under alternating conditions is substantially greater than the flow resistance.

To establish the variation of effective resistance with frequency the characteristic impedance $\left(Z_{0}\right)$ and the specific transmission constant $(\gamma=\alpha+j \beta)$ of a number of commonly used porous sound-absorbing materials have been measured over the range $100-1,300 \mathrm{c} / \mathrm{s}$ using samples 1 in. -3 in. thick inserted into a rigid tube $3.47 \mathrm{in.}$ in diameter. At each frequency sound is passed into the sample under two conditions in succession: first, when the sample is terminated by an impedance effectively infinite, and second when the terminal load is purely capacitive. By adopting an electroacoustic artifice the effect of the input impedance of the probe microphones, which measure the sound pressure, is completely eliminated, and by using an a.c. potentiometer to measure microphone voltages, the pressure ratio is derived as a complex number. A.ssuming that conventional transmission theory is applicable, this ratio is $\cosh (\gamma l)(l=$ sample thickness); whence $\gamma l$. Knowing $\gamma l$, the second test yields $Z_{0}$ in terms of the terminal capacitance.

After analysing the variation with frequency of these two parameters, it is found that in all the materials examined the series arms of the equivalent-tee network which represents the performance of the acoustical structure contain resistance and inductance in sories, the shunt arm being a capacitance in series with a small resistance. Where the test samples have been sufficiently stable to yield very precise data it has been possible to investigate the series arms of the tee network in detail; it is then found that each branch consists of two series combinations of $R$ and $L$ in parallel which have impedances $\left(R_{1}+j \omega L_{1}\right)$ and $\left(R_{2}+j \omega L_{2}\right), L_{2}$, say, being greater than $L_{1}$. Reanalysis of the data published in ref. 1 confirms this curious disposition. The same data also confirm the series arrangement of the shunt branch of the tee-network, a fact apparontly unrecognized by the authors. It is to be noticed that a later publication ${ }^{2}$ discusses the possibility of a rather similar disposition, using arguments of quite a different kind. Yet another publication ${ }^{3}$ presents data which on re-analysis confirm the series combination of $R$ and $C$, in the shunt arm, mentioned here.

If then we assume that the series arm of the equivalent te日-network has the dual form described, with $L_{2}>L_{1}$, it is clear that flow resistance measures the value of $R_{1}$ in parallel with $R_{2}$, whereas when frequency rises from zero the effective resistance rises towards $R_{1}$-a quantity which in various materials has been found to be of the order of 1.5 up to 3 or more times the flow resistance. Moreover, as $L_{2}$ may be many times greater than $L_{1}$, the frequency at which the transition from the lower to the higher effective resistance is substantially complete may be as low as $150 \mathrm{c} / \mathrm{s}$; usually it is in the region of $200-$ $300 \mathrm{c} / \mathrm{s}$.

Knowing both $Z_{0}$ and $\gamma$ it has been possible to go a stage further and to build electrical artificial lines, the input impedance of which imitates the acoustical impedance, at normal incidence, which sheets of any thickness of the tested materials would present when mounted on the walls of a room as sound absorbers. The real part of the electrical input impedance is-subject to the usual corrections - a measure of the acoustical resistance in which the sound would be absorbed. By modifying the length of the artificial line and its terminal impedance it becomes possible to predict the effect of altering the thickness of the absorber and of its method of mounting.

\section{E. R. WIGAN}

Research Department,

British Broadcasting Corporation, Kingswood Warren, Tadworth, Surrey.

1 Ferrero, M. A., and Sacerdote, G. C., Acustica, 10, 336 (1960). ${ }^{2}$ Kraak, W., Hochfrequenz Technik, "y1, No. 5 (October 1962).

${ }^{3}$ Esmail-Begui, Z., and Naylor, T. K., J. Acoust. Soc. Amer., 25, 87 (1953).

\section{Dielectric Properties of Cross-linked Polystyrene Film formed in the Glow Discharge}

IrRADIATION of a monomer vapour frequently causes polymerization leading to the formation of a solid deposit on any convenient surface. Electrons ${ }^{1}$, ultra-violet, $\mathrm{X}$-, $\gamma$-radiation or an electrical discharge ${ }^{2}$ can be used.

In the work recorded here. a glow discharge at $180 \mathrm{kc} / \mathrm{s}$ is used to form uniform $1 \mu$ polystyrene film. The discharge is maintained between parallel cylindrical aluminium electrodes in several $\mathrm{mm}$ mercury pressure of styrene vapour contained in a pre-evacuated chamber. Aluminium foil, in contact with one electrode, serves as the substrate for the film. This electrode rotates uniformly during polymerization. Aluminium is afterwards evaporated on to the surface of the polymer film to provide a second electrode.

Fig. 1 shows power factor and capacitance against temperature at several frequencies. The wide distribution of relaxation times, apparent from the shape of the curves, results from the complex structure of the film. Traces of oxygen and water vapour, which give rise to highly polar groups, are especially significant since only milligrams of polymer are formed. Further, electron spin resonance measurements made at room temperature in vacuo show the presence of free radicals which presumably peroxidize on exposure to air. 